\title{
Influence of the Heat Treatment on the Band Gaps in the Biopolymer Photonic Crystals
}

\author{
S. SaviC-Sevic*, D. Pantelic and B. Jelenkovic \\ Institute of Physics, Belgrade, Serbia
}

\begin{abstract}
1D photonic crystal was fabricated in a dichromate-sensitized biopolymer using holography method. The effects of the temperature of the photonic crystal layer on the center of photonic band gaps were studied. We showed that the central wavelength of the band gap can be tuned by heating the photonic crystal layer. By applying such heat treatment, tuning of the position of the band gap for about $80 \mathrm{~nm}$ was obtained.
\end{abstract}

PACS numbers: 42.70.Qs, 42.40Pa, 82.35.Pq, 87.15.rp

\section{Introduction}

Photonic crystals are dielectric materials that exhibit band gaps $(\mathrm{BG})$ in which electromagnetic wave propagation is forbidden [1]. Photonic crystals find various applications in single-mode waveguides [2], super-prisms [3], perfect lenses [4], and biosensors [5]. These applications would be significantly improved if the band structure of the crystal could be tuned.

In our laboratory we fabricate a one-dimensional dichromate biopolymer PC having a tunable photonic band gap. The biopolymer used in this study is pullulan. Pullulan is a natural linear polysaccharide, produced from starch by Aureobasidium pullulans. Upon heating the photonic crystal from the room temperature to $105^{\circ} \mathrm{C}$ we observed a wavelength shift of $80 \mathrm{~nm}$ for the wavelength band.

\section{Experimental}

Holographic photosensitive material was prepared by mixing $8 \%$ aqueous solution of biopolymer and 10\% ammonium dichromate by weight of biopolymer. The solution was coated onto clean glass slides in a horizontal position. Film was dried overnight under normal laboratory conditions. The thickness of the dried layer was only $\approx 10 \mu \mathrm{m}$. The thin layer ensures the narrower band gaps.

A single-frequency, diode pumped Nd-YAG laser, at $532 \mathrm{~nm}$, was used to generate PC in the holographic material. The laser beam was expanded and exposed biopolymer layer at normal incidence. Mirror was set behind the holographic plate. The hologram was obtained by interference of two oppositely directed beams inside the emulsion. The interference pattern consists of planes parallel to the substrate.

After exposure, plates were chemically processed. Processing involves washing the plates in mixture of water

* corresponding author; e-mail: savic@ipb.ac.rs and isopropyl alcohol (the ratio of water and alcohol in the bath was 1:1) for 120 seconds, and drying for 60 seconds in pure isopropyl alcohol.

The influence of temperature of the PC, and duration of the heat treatment on the position of the band gap were investigated. Transmission spectra of the white light from the halogen lamp, through biopolymer films were measured by the fiber-based spectrometer.

\section{Results}

Figure 1 shows the shifting of the transmission spectra of biopolymer photonic crystal during the process of heating the PC. The sample was heated from $25^{\circ} \mathrm{C}$ to $105^{\circ} \mathrm{C}$, in steps of $20^{\circ} \mathrm{C}$. Increasing the temperature of the sample we were able to shift the band gap position towards shorter wavelengths, from 561 to $481 \mathrm{~nm}$.

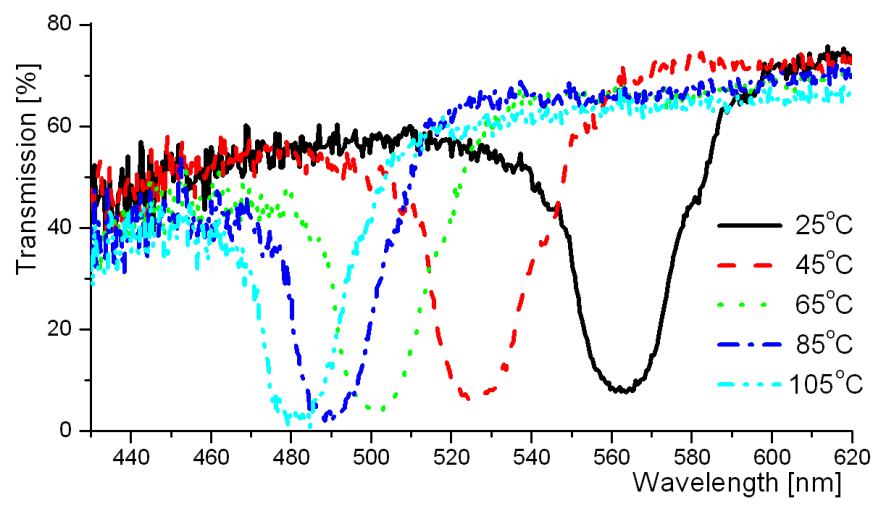

Fig. 1. Transmission spectra of the PC as a function of temperature. For measurements the halogen lamp and the fiber based spectrometer were used.

The total shift of the wavelength band gap as the temperature increases from 25 to $105^{\circ} \mathrm{C}$ degrees was about $80 \mathrm{~nm}$. We believe that the shift of the band gap position is due to temperature dependent refractive index of a biopolymer [6]. 
The changes of transmission spectra with prolonged heating at elevated temperature are shown in Fig. 2. The sample PC was placed on the plate held at $100{ }^{\circ} \mathrm{C}$ and transmission spectra are recorded in intervals of $15 \mathrm{~min}$. The band gap of the biopolymer PC at room temperature $\left(25^{\circ} \mathrm{C}\right.$ ) is centered at $561 \mathrm{~nm}$ (solid line in Fig. 2). After first 15 minutes at $100^{\circ} \mathrm{C}$ the shift of the center of the wavelength band gap was from $561 \mathrm{~nm}$ to $481 \mathrm{~nm}$, i.e. about $80 \mathrm{~nm}$. It can be seen from Fig. 2 that there are no further significant changes of the band gap position, with prolonged heat treatment.

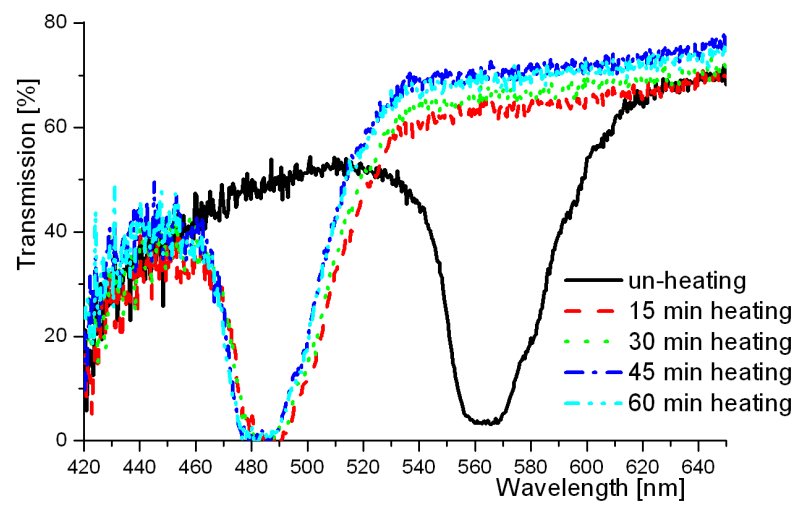

Fig. 2. Transmission spectra as a function of heat treatment duration.

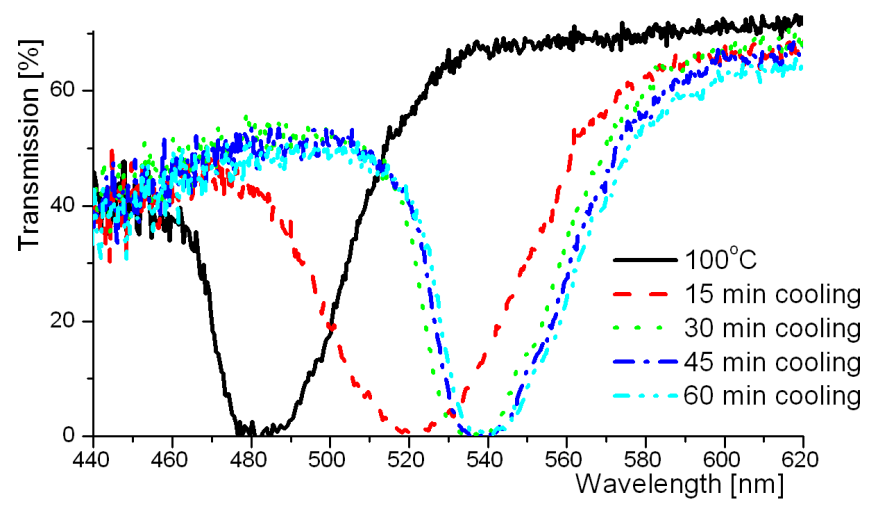

Fig. 3. Transmission spectra as a function of cooling duration.

Next, the sample was left to cool down to room temperature and transmission spectra were measured for a period of one hour, in intervals of $15 \mathrm{~min}$. Figure 3 shows the transmission spectra of biopolymer photonic crystal measured during the cooling process. With temperature decreasing from $100^{\circ} \mathrm{C}$ to a room temperature, the band gap position shifts back towards longer wavelengths from 481 to $540 \mathrm{~nm}$.

\section{Conclusion}

In conclusion, we have developed PC, using dichromate biopolymer, with tunable photonic band gap. The center of the wavelength of the PC was shifted over $80 \mathrm{~nm}$ towards the lower wavelength by raising the temperature from room temperature to $105^{\circ} \mathrm{C}$. The changes of the location of the band gap occur fairly quickly after the PC is heated to the new temperature. Cooling the PC shifts the center of the band gap back to the longer wavelength which shows reversibility of the tuning process. These results are important as they present an easy way to move the center wavelength of band gap of the PC, thus making a tunable filter at optical frequencies.

\section{Acknowledgments}

The work has been done within the project OI 141003 financed by the Ministry of Science and Technological Development, Republic of Serbia.

\section{References}

[1] K. Busch, S. Lolkes, R.B. Wehrspohn, F. Foll, Photonic Crystals. Advance in Design, Fabrication and Characterization, Wiley-VCH, Weinhem 2003.

[2] A. Mekis, J.C. Chen, I. Kurland, F. Shanhui, R. Pierre, Villeneuve, J.D. Joannopoulos, Phys. Rev. Lett. 77, 3787 (1996).

[3] T. Prasad, V. Colvin, D. Mittleman, Phys. Rev. B 67, 165103 (2003).

[4] P.V. Parimi, T.L. Wentao, V. Plarenta, S. Sridhar, Nature 426, 404 (2003).

[5] W. Zhang, N. Ganesh, I.D. Block, B.T. Cunningham, Sensors Actuators B 131, 279 (2008).

[6] W. Belhadj, O. Boukari, D. Gamra, F. AbdelMalek, H. Bouchriha, Synthetic Metals 151, 6 (2005). 